\title{
Multiscale Characterization of Chronobiological Signals Based on the Discrete Wavelet Transform
}

\author{
F. H. Y. Chan*, Member, IEEE, B. M. Wu, Student Member, IEEE, F. K. Lam, Paul W. F. Poon, and A. M. S. Poon
}

\begin{abstract}
To compensate for the deficiency of conventional frequency-domain or time-domain analysis, this paper presents a multiscale approach to characterize the chronobiological time series (CTS) based on a discrete wavelet transform (DWT). We have shown that the local modulus maxima and zero-crossings of the wavelet coefficients at different scales give a complete characterization of rhythmic activities. We further constructed a tree scheme to represent those interacting activities across scales. Using the bandpass filter property of the DWT in the frequency domain, we also characterized the band-related activities by calculating energy in respective rhythmic bands. Moreover, since there is a fast and easily implemented algorithm for the DWT, this new approach may simplify the signal processing and provide a more efficient and complete study of the temporal-frequency dynamics of the CTS. Preliminary results are presented using the proposed method on the locomotion of mice under altered lighting conditions, verifying its competency for CTS analysis.
\end{abstract}

Index Terms-Characterization, chronobiological signals, tree structure, wavelet maxima, wavelet transform, zero-crossings.

\section{INTRODUCTION}

B IOLOGIC clocks are self-sustained internal timing mechanisms of all living organisms. Long-term disruption of the biological rhythms can cause health problems in human. Mechanisms of the biological rhythms are still not well understood. Further study is necessary to characterize the biological clocks and to allow intervention when they go awry.

In practice, it is difficult to study the biological clocks directly. Often the explicit behavioral activities are studied over time, called chronobiological time series (CTS) [1]. Since CTS is the result of different underlying clocks, it is a complex signal, consisting of many periodic components. From the dynamic system point of view, CTS is considered chaotic, and, regarding temporal statistical characteristics, nonstationary [1], [2]. Thus, it is hard to derive mathematical models for them. Most chronobiological studies concentrated on activity at 24-h periodicity (circadian rhythm). In contrast, higher-frequency rhythms were

Manuscript received September 29, 1997; revised June 29, 1999. This work was supported in part by grants from the Hong Kong Research Council and in part by the National Science Council under Grant NSC86-2314-B006-096. Asterisk indicates corresponding author.

*F. H. Y. Chan is with the Department of Electrical and Electronic Engineering, The University of Hong Kong, Hong Kong HKSAR (e-mail: fhychan@eee.hku.hk).

B. M. Wu and F. K. Lam are with the Department of Electrical and Electronic Engineering, The University of Hong Kong, Hong Kong, HKSAR.

P. W. F. Poon is with the Department of Physiology, School of Medicine, National Cheng Kung University, Tainan, 70101, Taiwan.

A. M. S. Poon is with the Department of Physiology, The University of Hong Kong, Hong Kong, HKSAR.

Publisher Item Identifier S 0018-9294(00)00244-5. overlooked [3], due to its complexity and the lack of suitable analysis methods.

Approaches used for CTS analysis fell into two categories: 1) frequency- and 2) time-domain. For frequency-domain, Fourier transform was widely used [4], [5]. In addition, modern spectrum estimation techniques [6], such as autoregressive (AR), autoregressive and moving average (ARMA), autocorrelation and Cosinor analysis [7] were often adopted. Because of the assumption of stationarity, these methods provided minimal information regarding the temporal dynamics. An improved technique in the time domain, called complex demodulation (CD), has been developed recently [2] to extract the temporal regularity (i.e., amplitude and phase) of constituent frequencies, yielding a smooth function (i.e., remodulate function) associated with each frequency. Other approaches assume nonlinear dynamic system properties, and their results are difficult to interpret.

The wavelet transform, (WT) [8], [9]-[13] makes a good compromise between time and frequency resolutions. It decomposes a signal into a time-frequency or time-scale plane, showing simultaneously its time-frequency behavior, and its time-frequency (or scale) localization is higher than the short-time fast Fourier transform (SFFT). Recently, we [3], [14] have used the Morlet continuous wavelet transform (CWT) to study the locomotion rhythms of animals, visualizing ultradian rhythms for the first time. However, representations of the CWT in the time-scale plane are often blurred and interpretations still difficult. As part of a continuing effort to develop more efficient and effective methods for CTS analysis [15], we propose in this paper a new approach based on a particular DWT developed by Mallat [16], [17]. Using edge-detection algorithms [18], [19], we extracted the wavelet maxima (which correspond to points of sharp variations or edges) in the signal for further analysis. Though wavelet maxima have been successfully used in various image and signal processing tasks [20]-[22], image classification [23] and electrocardiogram analysis [24], [25], such technique has not yet been applied in this way to chronobiology.

We expanded the wavelet maxima representation by extracting both the wavelet maxima and zero-crossings of the wavelet signals and used them to characterize the rhythmic activities. A tree scheme was further constructed to organize those interacting activities for easy visualization. Since this DWT is equivalent to a series of bandpass filters in the frequency domain, activity intensity of rhythmic activities can be examined by the energy profile in the rhythmic bands.

To test the efficiency of the proposed method, an experiment was carried out to study the synchronizing ability of mouse locomotion to an artificial dark-light (DL) reversal or light tran- 
sition. Results verified the competency of the present method over CWT and CD.

\section{The WAVELET TRANSFORM}

The well-known WT decomposes a signal into components appearing at different scales [11]-[13], [16]. Mallat et al. constructed a family of wavelets in such a way that they are $p$ th order derivatives of a smoothing function [16], [17] and, further, gave a reduced representation of the wavelet signals, the wavelet maxima representation, and showed that a signal could be reconstructed precisely with stability based on its wavelet maxima [26]. For a $J$-scale WT, the wavelet maxima representation of a signal is defined as $\left\{S_{2^{j}} f(x),\left[M_{2^{j}}\right]_{1 \leq j \leq J}\right\}$, where $M_{2^{j}}=\left\{\left(x_{i}, W_{2^{j}} f\left(x_{i}\right)\right):\left|W_{2^{j}} f(x)\right|\right.$ has a local maximum at $\left.x=x_{i}\right\}$ is the wavelet maxima set and $S_{2^{J}} f(x)$ is the approached signal at the coarsest scale $2^{J}$. It may be noted that $S_{2^{j}}$ is a smoothing operator at scale $2^{j}$ and $S_{2^{0}} f(x)$ is the original signal. In other words, the $J$-level wavelet maxima representation of a signal contains positions of $W_{2^{j}} f(x)$ at all levels where $\left|W_{2^{j}} f(x)\right|$ attains a local maximum, plus the smoothed signal at the coarsest scale. The coarsest scale approaching signal $S_{2^{J}} f(x)$ is included to ensure the completeness of a finite-scale representation. For details of the description of the wavelet maxima representation and the efficient implementation of the reconstruction algorithm, readers can refer to the original work [27].

As CTS is the result of different underlying clocks of many periodic components, WT provides an effective method of tracking them. The WT of the CTS at successive scales is equivalent to bandpass filtering at successive narrowing bandwidths so that details of different rhythmic activities of the CTS can be explored simultaneously. By virtue of its maxima representation capability as well as its multiscale analysis feature, and the bandpass filtering nature, WT is expected to provide a more powerful tool for chronobiology study, with special reference to its dynamics.

\section{METHODS}

\section{A. Experimental Methods}

Locomotion is an important aspect of chronobiological behavior. Locomotion of either animal or human responds to the day-night cycle as introduced by an artificial light-dark (LD) schedule, shift work or jet lag. Such changes have been studied only in circadian rhythms using actograms, and generally considered, without proof, that one week is necessary for complete adaptation to the new lighting environments. In this experiment we intend to give a more thorough study of animal locomotion using a new approach.

1) Data Acquisition: Male mice (Strain C57, aged 8-10 weeks) were used. The locomotor activity of each mouse was studied with our image-based system developed [3], [28]. In brief, a mouse was individually housed in a top-open perspex cage with normal food and water supply put in a sound-shield chamber (IAC) with ventilation and light control. The displacement of the animal was detected by summing the pixels in the difference image of two consecutive frames $2 \mathrm{~s}$ apart. To study the ability of mice to synchronize with external lighting

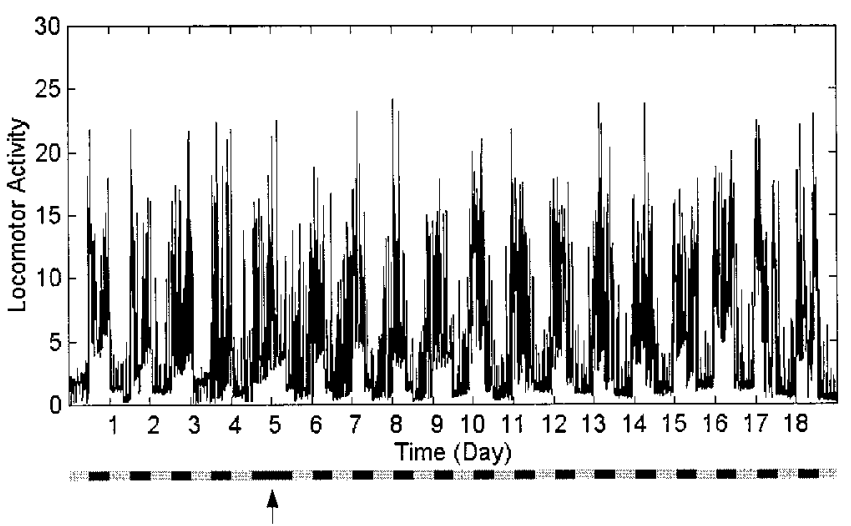

Fig. 1. A representative example of the locomotor activity time profile of a mouse recorded over 18 days. The DL phases are indicated by the dark-gray bars at the bottom. A DD light transition (arrow) was introduced on day five.

conditions, each experimental session lasted for a total of 18 days with a DL cycle (12 h: $12 \mathrm{~h}$ ) given in the first four days, and then followed by a reversal LD cycle by inserting a $12-\mathrm{h}$ dark-dark (DD) transition on day five. For simplicity, we called the first four days the control period, and the following 14 days the functional period. A total of four mice were studied.

2) Preprocessing: Empirically we found that the most consistent features in the CTS are below 100 cycles per day (cpd). Therefore, we only focused on circadian and ultradian rhythms. A coarse resolution locomotor signal $L$ was then extracted from the raw data by computing the root mean square values over time windows of 7.2-min (equivalent to $100 \mathrm{cpd}$ ). Fig. 1 gives a representative example of the locomotor activity of a mouse according to the lighting conditions described in last section. The preprocessed locomotor signal was then subjected to wavelet analysis as described below.

\section{B. Characterization of Locomotor Activities}

In the following, we present the new approach for CTS analysis. It should be mentioned that although the methodology of the new approach was derived from the locomotor activity, it can be applied to other CTS without loss of generality.

1) Definition of the Locomotor Activities: In the present study, seven scales were chosen, corresponding to the seven rhythmic bands of biological rhythms [7]. Scale $2^{7}$ corresponds to the circadian rhythms (around $1 \mathrm{cpd}$ ), scales $2^{6}$ and $2^{5}$ to the intermediate rhythms range $(2-5 \mathrm{cpd})$, scale $2^{4}$ is related to subultradian rhythms $(5-11 \mathrm{cpd})$, scale $2^{3}$ corresponds to the ultradian rhythms $(12-20 \mathrm{cpd})$, and scales $2^{2}$ and $2^{1}$ to the supraultradian rhythms (21-29 cpd) and very high-frequency rhythms (more than $30 \mathrm{cpd}$ ). Thereafter, from the wavelet signals $\left(W_{2^{j}} L(x)\right)_{1 \leq j \leq 7}$ at seven scales, the wavelet maxima were extracted and the wavelet maxima representation $\left\{S_{2^{7}} L(x),\left[M_{2^{3}}\right]_{1 \leq j \leq 7}\right\}$ constructed. Fig. 2(a) and (b) gives, respectively, the $\mathrm{WT}$ and wavelet maxima representation of the signal shown in Fig. 1. From Fig. 2(b) it is clear that the sharp variation points of the signal can be characterized by the wavelet maxima.

We noted that besides the wavelet maxima, there were other interesting features in the wavelet signals, like zero-crossings; we, therefore, extracted the zero-crossings in each of the wavelet 


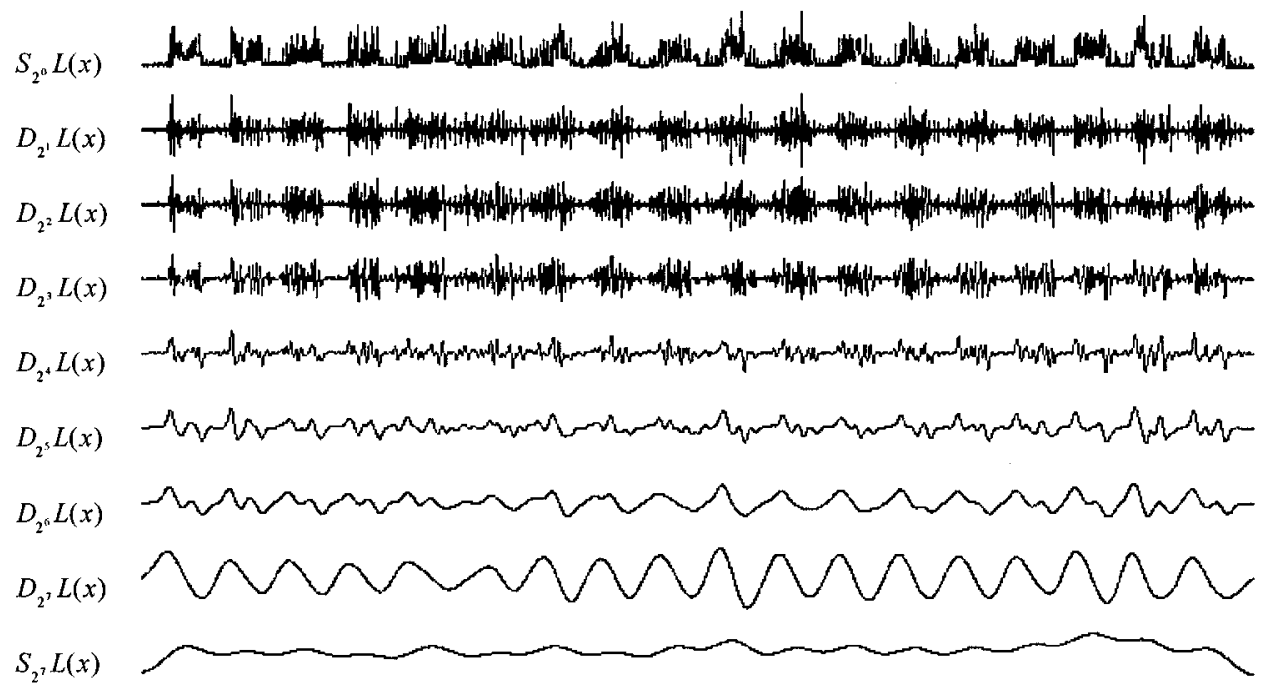

(a)

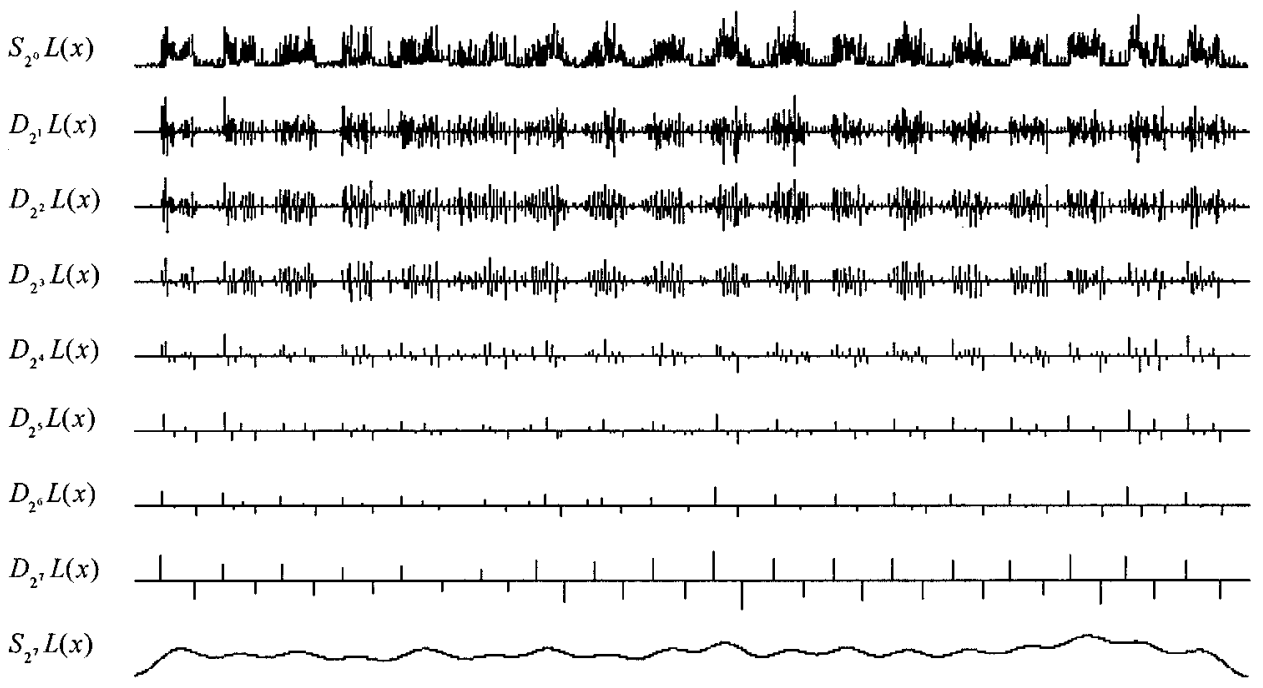

(b)

Fig. 2. (a) The WT of the signal shown in Fig. 1. (b) The wavelet maxima representation of the same signal. Each vertical line indicates the position and amplitude of a modulus maximum.

signals. As the signals were discrete, we had to adopt a particular convention to define a zero-crossing. In the case of a negative-valued sample followed by a positive-valued sample, the zero-crossing was defined as a position of the positive-valued sample. In the case of a positive-valued sample followed by a negative-valued sample, the zero-crossing was defined as the position of the negative-valued sample. Then the following rules were applied to relate the start, end and peak of an activity and the border between two neighboring activities to the wavelet maxima and zero-crossings.

1) A positive maximum indicates a start of an activity.

2) A negative minimum indicates an end of an activity.

3) A zero-crossing following a positive maximum and before a negative minimum indicates a peak of an activity.

4) A zero-crossing before a positive maximum and following a negative minimum indicates the position of a border between two activity peaks.
After analyzing all the $\left(W_{2^{j}} f(x)\right)_{1 \leq j \leq 7}$ signals, we obtained sets of parameters $\left\{s_{i}^{j}, A_{s_{i}}^{j}, P_{i}^{j}, e_{i}^{j}, A_{e_{i}}^{j}, b_{i}^{j}\right\}_{1 \leq j \leq 7}, i=$ $0, \cdots, N^{j}$, where $N^{j}$ is the number of activities at a given scale. Each set of these parameters characterizes the locomotor activities at a given scale in terms of the positions of start, peak, end and border (i.e., $s_{i}^{j}, P_{i}^{j}, e_{i}^{j}, b_{i}^{j}$ ) and the amplitude values of the start and end points (i.e., $A_{s_{i}}^{j}, A_{e_{i}}^{j}$ ). Fig. 3 illustrates how these parameters define a locomotor activity at a given scale.

2) Tree Representation: With the above scheme, locomotor activities at respective scales can be fully characterized. However, they are still too loosely structured, and the interactions between activities at different scales are not visualized. There is a need for higher level representations that characterize these temporal-scaling interactions more explicitly. It is known that the tree structure is most suitable for organizing signals with different sizes. Lu et al. [29] constructed a wavelet maxima tree for noise reduction. In this study, we proposed a tree description for the locomotor activity signal, called activity tree, and 


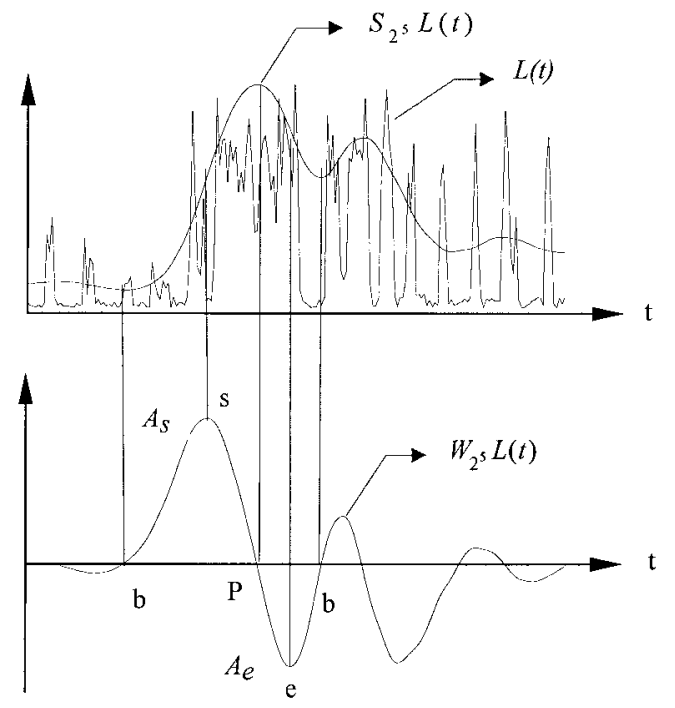

Fig. 3. Illustration of the parameters that define a locomotor activity at one scale (here $2^{5}$ ). The different curves are the original signal $L(t)$, the coarse approximation $S_{2^{5}} L(t)$ and the wavelet coefficients signal $W_{25} L(t) . s, e$, and $P$ represent, respectively, the time positions of the start, end, and peak. $A$ represents the amplitude values of the wavelet maxima at start and end points.

furthermore gave a "realistic" visualization of the activity tree, taking advantage of the multiscale information.

Before constructing the activity tree, we first defined the following terms.

a) Level: Each level of an activity tree corresponds to one level of scale or resolution. The coarsest scale in the DWT defines the root level of the tree, and the finest scale defines the tip-end level.

b) Node: The activities at a certain scale are nodes at that level of the tree.

c) Parent-Child Pair: Nodes that are linked at neighboring levels form a set of parent-child pairs. The node at the coarser scale is called the parent node and those at the finer scale are called child nodes. A parent-child pair between neighboring levels is identified if interactions exist between the nodes. A quantitative measure of interactions between nodes at neighboring levels is to be defined in the latter part of this section. By the causality assumption, a parent node must have at least one child node.

d) Branch: A branch is a path from a node to the tip-end of the tree. There may be different branches from one node to the tip-end of the tree.

Thus, the tree description is a hierarchical data structure. Each node in the tree has several attributes: level, positions, values, parent node, child nodes, and the branch to which it belongs. The first three attributes have been included in the parameter sets given above. The other three attributes are unique for characterizing the relationship between activities.

Construction of the activity tree consists of a procedure of corresponding, weighting and linking all related activities at neighboring scales.

e) Corresponding: Since by increasing the resolution the number of activities would also increase, there is generally no one-to-one correspondence between two successive scales. Nevertheless, we noted that the large activities at coarser scales merged with the small activities at finer scales. Moreover, there were no spurious details generated by the WT when passing from fine to coarse scales. So the above ambiguity can be solved by applying a minimum distance criterion, that is, two activities $A_{m}^{j+1}$ and $A_{n}^{j}$, respectively, at scales $2^{j+1}$ and $2^{j}$ are in correspondence if their peak positions satisfy

$$
\begin{gathered}
\operatorname{distance}\left(\mathrm{t}_{\mathrm{P}_{\mathrm{A}_{\mathrm{m}}}}^{\mathrm{j}+1}, \mathrm{t}_{\mathrm{P}_{\mathrm{A}_{\mathrm{n}}}}^{\mathrm{j}}\right)=\min \left\{\operatorname{dist}\left(t_{P_{A_{m}}}^{j+1}, t_{P_{A_{i}}}^{j}\right)\right\}, \\
i=0, \cdots, N^{j+1} .
\end{gathered}
$$

We started the corresponding procedure at the finest scale $2^{1}$. By repeatedly applying the minimum distance corresponding criterion, the parent-child node pairs at successive scales were established. Furthermore, the parent-child relationship was included into the parameter sets of both parent and child nodes.

f) Weighting: In order to facilitate the identification of the main activities, we weighted the interacting intensity of each parent-child pair according to the contribution in activity made by each child with its parent activity. We calculated the energy of each child activity, and then computed the ratio of energy in each child node to the energy in the parent node and used it as an indication of the interaction between each parent-child pair.

g) Visualizing: To provide a visual overview of the temporal-scaling structure and the intensity of the locomotor activity, we built a "realistic" tree following the corresponding and weighting steps. This was achieved by defining the nodes of the "realistic" tree at the peak points, and linking all parent-child pairs using different gray-levels or pseudocolor line segments according to individual interacting intensities. At this stage, the (weighted) activity tree was obtained, and the main activity branches could be readily identified.

It is obvious that given a tree representation, the activities and their temporal-scaling relations are fully characterized. The tree branches convey important information on interaction between activities that is absent in the raw WT and wavelet maxima representation. From the tree branches, we could easily see the number of child activities included in each parent activity, the interacting intensity between each parent-child pair, and the whole activity pattern in the CTS. Additionally, since the scale $2^{7}$ (root level of the tree) is related to daily activity, the above construction procedure gives a natural segmentation of daily activities, which is really desirable in examining daily changes of the locomotor behavior of mice around the shifting of lighting conditions. Fig. 4 gives the result of the tree representation of the signal shown in Fig. 1.

Visual inspection of the activity tree may, thus, add new information on the recovery process of locomotor activity and lead us to the next analysis. Based on the tree characterization, we studied the synchronizing ability of mice to the light transition as presented in the following section.

\section{Synchronizing Ability of Mice to the Light Transition}

1) Transition Patterns: To study the ability of mice to synchronize to the light transition, we focused our analysis on temporal changes in locomotor activity induced by the LD reversal. We first aligned the daily activity at each scale. Since the activity tree representation has divided the locomotor activity time series into a set of daily activity segments, we simply aligned the 


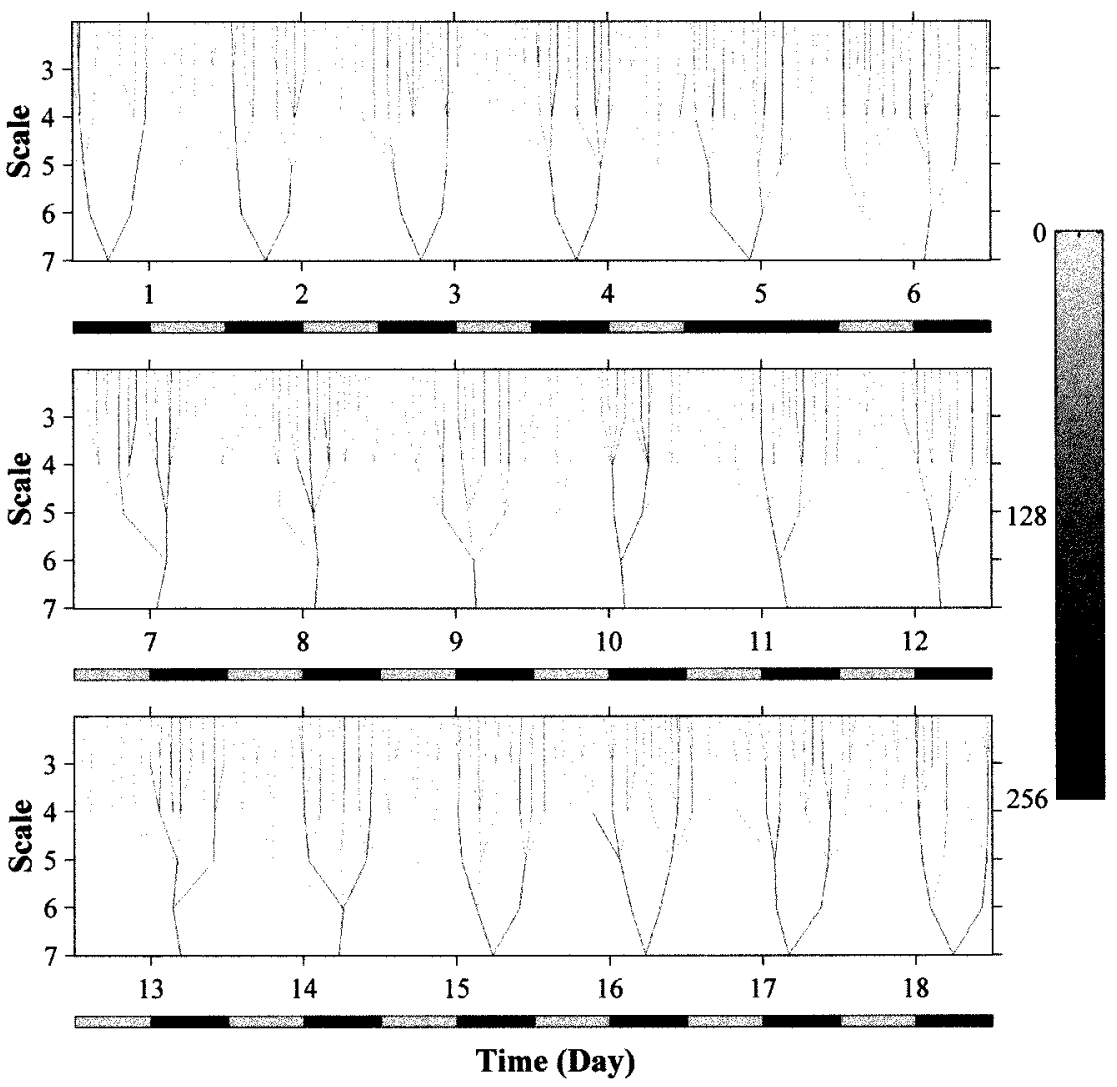

Fig. 4. A representative example of the daily activity trees extracted from the locomotor activity time profile of a mouse (same data as Fig. 1), with a DD transition introduced on day five. Branches from scale $2^{7}$ to $2^{2}$ are shown. Note at scale $2^{6}$, there is change from double branches to a single branch pattern on day seven to day 14 , and then back to double branches ("2-1-2" pattern). See text for details.

time location of the start, peak, and end points of each daily segment at respective scales according to the lighting schedule of that day. By this method, the transition patterns of different rhythmic activities can be readily identified and subjected to further analysis.

It must be pointed out that in the conventional actogram plot, the original locomotor signal is commonly aligned. Since the circadian activity is much greater in amplitude than the high-frequency activities, the transition of these small activities might be concealed by the larger circadian activity. On the other hand, our feature-points-based multiscale alignment works at individual scales and, thus, reveals the underlying patterns more clearly.

2) Statistical Analysis: As can be seen from the activity tree representation (Fig. 4), there were day-to-day variations of the locomotor activity, in particular at fine scales. Statistical analysis was carried out as follows. First, the ensemble average across animals was used to suppress intersubject variations. In the present study, for large activities at coarse scales, we focused our analysis on the time location of the start, peak, and end points. Given the more complex activity pattern at finer scales, no apparent transition patterns could be observed by aligning the plots. Thus, we turned to study activity-related parameters such as the length of the active period or the average activity time. The cumulative energy was also used as an overall index of the intensity of activity in various rhythmic bands. To further facilitate the observation of daily variations induced by the al- tered LD schedule, we calculated the cumulative energy in each rhythmic band.

From the activity tree, the following parameters were extracted to give a detailed picture on the synchronizing ability of mice to the altered LD transition.

1) The average time location of the start, peak, and end points at scale $2^{7}$.

2) The average time differences between the start and peak points $\left(\Delta t_{1}\right)$ and between the peak and end points $\left(\Delta t_{2}\right)$, and then the activity time $\left(\Delta t_{1}+\Delta t_{2}\right)$. Particularly, the $\left(\Delta t_{1}+\Delta t_{2}\right)$ at scale $2^{7}$ was defined as the daily activity time.

3) The cumulative energy in each rhythmic band.

3) Time Constant: The transitional pattern at scale $2^{7}$ was close to an exponential function, thus allowing approximation by an exponential equation

$$
H(d)=\Delta h\left(1-e^{-d / \tau}\right)
$$

where

$\tau$ time constant;

$\Delta h \quad$ time shift of the circadian activity;

$d \quad$ time unit in days.

In the present study, with a phase shift $(\Delta h)$ of $12 \mathrm{~h}$, the time constant of the exponential function was found by the least mean square criterion. Thereafter, the time shift at any day after the introduction of the dark-light transition can be estimated. 


\section{RESUlTS}

Based on the daily activity trees (Fig. 4), the day-to-day evolution of the locomotor activity, including both the temporal-scaling structure and the intensity, can be better revealed compared to its representation by WT [Fig. 2(a)] and wavelet maxima [Fig. 2(b)]. Before the DD light transition introduced on day five, the activity trees appeared quite similar across days. Each daily tree consisted of two main activity branches (starting from scale $2^{6}$ ) that emerged during an early and a late part of the dark phase. This is consistent with the presence of two activity bouts in the night as reported previously for the same animal [3]. For simplicity, we called this a "double peak" activity pattern. After the DD light transition, especially during the first two days, the high-frequency rhythmic activities increased in both the number of activities (number of branches) and the daily activity time (width of tree) as well as a change in the symmetry of the tree pattern. Thereafter, it switched to a single peak activity pattern and persisted till day 15 (i.e., ten days after the DD light transition). By visual inspection of the tree representation, an evolution of locomotor tree activity from double to single, and then back to double pattern was most apparent. For simple descriptive purposes, we called this "2-1-2" pattern.

Fig. 5 shows the peak location at scale $2^{7}$ relative to the $24-h$ period that was shifted by the DD light transition. Data were averaged from four animals over a period of 18 days with a DD light transition introduced on day five. For easy visualization of the curve fitting, the $y$-axis was intentionally formed by extending the 24-h period to a two-day period and displayed the relevant time range up to $35 \mathrm{~h}$ (we called this the "relative time"). Prior to light transition, the peak location was stable in time (occurring around 19th h of each day). Following the DD transition, we expect to find a peak shift equivalent to $12 \mathrm{~h}$. This, indeed, happened over a period of almost ten days. As the new peak location fell toward the thirty-first hour, corresponding to the seventh hour of the next day (or a 12-h shift from the nineteenth hour). In this plot, the transitional effect was approximated by an exponential function (Fig. 5, solid line) with a time constant of 3.91 days. This allowed for the first time, a quantitative estimation of the time required to adjust the rhythmic pattern back to its control level. For this animal, a maximal time shift of $12 \mathrm{~h}$ would require 12 days to return to $95 \%$ of the pretransitional pattern.

The result is also consistent with other aspects of the transitional change manifested in the alteration in daily active time (i.e., the number of hours per day the animal was active in the cage). The average time profile ( $n=4$ animals) is shown in Fig. 6. Right after the transition on day five, daily active time markedly increased during the first three days and then slightly depressed before returning back to the pretransitional level around day 18. The change in total activity was however less pronounced than the tree representation.

We processed the same datasets using the CD and CWT approaches for a brief comparison. We used a software package (WINCD) [30] for the CD approach developed by the Office of Military Performance Assessment Technology, and the result is consistent with our present method, but the CD method

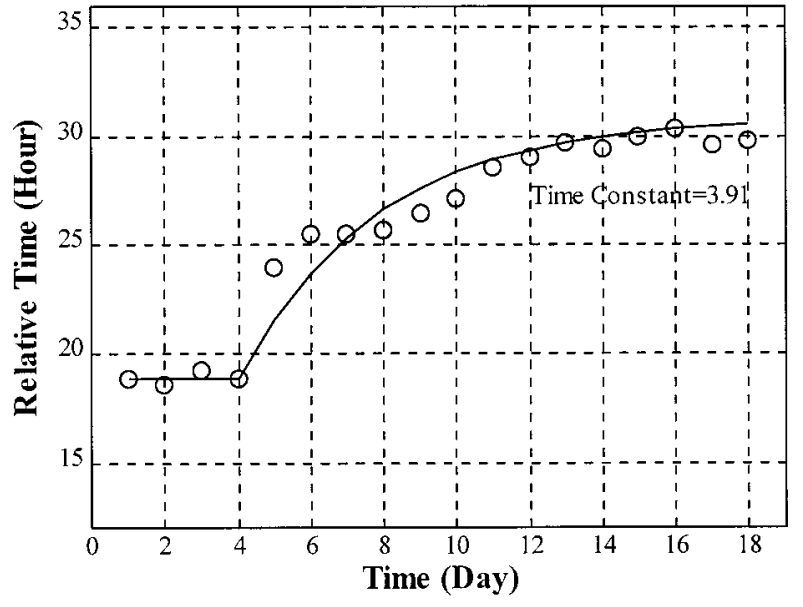

Fig. 5. The temporal shift in the daily branch location (scale $2^{7}$ ) after a DD light transition introduced on day five (open circles, average of four animals). The shift was fitted with an exponential function (solid line). "Relative time" marks the location of the peak with respect to each day (up to $24 \mathrm{~h}$ ). For display purposes, two consecutive days were put together (up to $48 \mathrm{~h}$ ). Only the relevant time range from 15 to $35 \mathrm{~h}$ was displayed. See text for details.

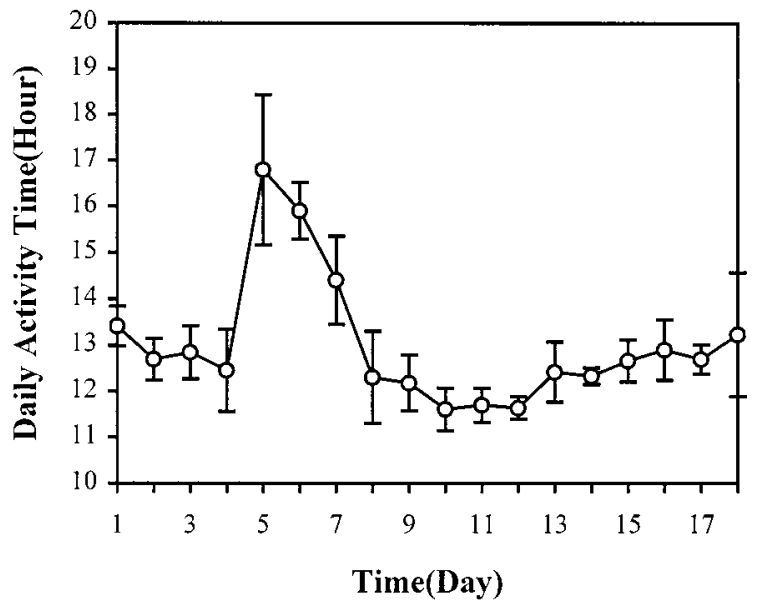

Fig. 6. Average daily activity time ( $n=4$ animals, showing mean and SD) with a DD light transition on day five. Note the initial fast rise, and the subsequent slight depression and gradual recovery to control level toward the end.

has some difficulty in determining the day-to-day adaptation of different rhythmic activities. We also used the Morlet wavelet in the CWT approach to calculate the time-frequency plot. The results of the CWT is far more difficult to use since they are not decomposed into frequency bands as in the discrete wavelet case. Our new method is also more computationally efficient when compared to the CD or CWT methods.

\section{CONCLUSION AND DisCUSSION}

In the present study we have developed a novel approach, the first attempt in the literature to analyze CTS using feature points from the DWT. The approach was tested on motor activity of a small rodent in synchronization to DL transitions and successfully quantified the transient changes of different rhythms, the evolution of temporal-scaling structure and activity energy changes. Preliminary results suggested that this is 
a better method in comparison with CD and CWT for the CTS analysis.

The activity tree we used has organized the temporal-scaling interactions of different rhythmic activities in the CTS and allows easy visualization of the evolution of both the temporalscaling structure and its intensity changes. With the activity tree, previously unreported aspects of the locomotion were revealed, e.g., the "2-1-2" activity evolution pattern. Results indicated that the DL transition may disrupt not only the intensity of activity, but also the fine activity patterns. The physiological implication of this new phenomenon however remains to be studied. The day-to-day and intersubject variations of the locomotor activities, in particular at fine scales, may be due to the intrinsic property of the biological rhythms, the intersubject variations as well as the possible fluctuation caused by slight changes in environmental conditions not being noticed. From the energy profiles, we further found a time delay in the response to the DL transition between the high- and low-frequency rhythmic locomotor activities. The physiological mechanisms of these results could well be related to the multiple oscillators postulated to exist in the body [3]. At this stage, we only examine the results in a semiquantitative way prior to any effective criterion can be developed to evaluate the similarity of activity trees.

While there are many discrete wavelets to choose from, we used the spline wavelet primarily because of its shift-invariant property. Hence, a translation in the signal will cause the zerocrossings and local extrema in the WT to be translated by the same amount. Therefore, it should be more appropriate for our study of dynamics. Besides the presently used quadratic spline wavelet, we also tested higher order spline wavelets. The equivalent filter bandwidth of these wavelets is similar to that of the quadratic spline. And their results are almost identical. Since the quadratic spline wavelet has a shorter coefficient series and is, therefore, a good choice for fast computation.

This research represents an effort toward developing more effective and efficient approaches for CTS analysis and also shows the potential of the WT and activity trees, especially for processing time-varying biomedical signals. The power of WT lies in its multiscale information analysis which can fully characterize a signal.

\section{ACKNOWLEDGMENT}

The authors wish to thank Y. K. Pang and K. K. Wing for their technical help and Dr. T. F. Elsmore (Activity Research Services, 10284 Perez Court, San Diego, CA 92124) for providing the WINCD software package for our comparative study.

\section{REFERENCES}

[1] J. D. Prins and B. Hecquet, "Data processing in chronobiological study," in Biologic Rhythms in Clinical and Laboratory Medicine, Y. Touitou and E. Haus, Eds. Berlin, Germany: Springer-Verlag, 1992, pt. III, pp. 90-113.

[2] D. P. Redmond and H. C. Sing, "Biological time series analysis using complex demodulation," in Rhythmic Aspect of Behavior, F. M. Brown and R. C. Graeber, Eds. Hillsdale, NJ: Lawrence Erlbaum Assoc., 1982, ch. 12, pp. 429-457.

[3] A. M. S. Poon, B. M. Wu, P. W. F. Poon, E. P. W. Cheung, F. H. Y. Chan, and F. K. Lam, "Effect of cage size on ultradian locomotor rhythm of laboratory mice," Physiology and Behavior, vol. 62, pp. 1253-1258, 1998.
[4] Cambas and T. Diez-Noguera, "Evolution of rat motor activity circadian rhythm under three different light patterns," Physiology \& Behavior, vol. 49, pp. 63-68, 1991.

[5] J. Beau, "Activity rhythms in mice-III: Stability and plasticity of rhythm characteristics in experimental and environmental conditions," Physiology \& Behavior, vol. 52, pp. 231-235, 1992.

[6] S. B. Kesler, Modern Spectrum Analysis, II. Piascataway, NJ: IEEE Press, 1986.

[7] C. Bingham, B. Arbogast, G. C. Guillaumc, J. K. Lee, and F. Halberg, "Inferential statistical methods for estimating and comparing cosinor parameters," Chronobiologia, vol. 9, pp. 397-439, 1982.

[8] A. Grossmann and J. Morlet, "Decomposition of Hardy functions into square integrable wavelets of constant shape," SIAM J. Math., vol. 15, pp. 723-736, 1984

[9] S. Mallat, "Multifrequency channel decompositions of images and wavelet models," IEEE Trans. Acoust. Speech Signal Processing, vol. 37, pp. 2091-2110, Dec. 1989.

[10] S. Mallat, "A theory for multiresolution signal decomposition: The wavelet representation," IEEE Trans. Pattern Anal. Machine Intell., vol. 11, pp. 674-693, July 1989.

[11] I. Daubechies, "The wavelet transform, time-frequency localization and signal analysis," IEEE Trans. Inform. Theory, vol. 36, pp. 961-1005, Sept. 1990

[12] Y. Meyer, Wavelets and Operators. Cambridge, U.K.: Cambridge Univ. Press, 1992.

[13] I. Daubechies, Ten Lectures on Wavelets. Philadelphia, PA: SIAM, 1992.

[14] B. M. Wu, F. H. Y. Chan, F. K. Lam, A. M. S. Poon, P. W. F. Poon, and D. C. S. Chow, "Characterization of the chronobiological signals based on the continuous wavelet transform," in Proc. IEEE-BMES Conf., Chicago, IL, 1997, pp. 1620-1623.

[15] B. M. Wu, F. K. Lam, F. H. Y. Chan, P. W. F. Poon, and A. M. S. Poon, "Multiscale analysis of activity rhythms of animals," in Proc.IEEE-EMB'95, 1995, pp. 1085-1086.

[16] S. G. Mallat and S. Zhong, "Characterization of the signals from multiscale edges," IEEE Trans. Pattern Anal. Machine Intell., vol. 14, pp. 710-732, July 1992.

[17] S. G. Mallat and W. L. Hwang, "Singularity detection and processing with wavelets," IEEE Trans. Inform. Theory, vol. 38, pp. 617-643, Mar. 1992.

[18] D. Marr and E. Hildreth, "Theory of edge detection," in Proc. Roy. Soc. Lond., vol. B-207, 1980, pp. 187-217.

[19] J. Canny, "A computational approach to edge detection," IEEE Trans. Pattern Anal. Machine Intell., vol. PAMI-8, pp. 679-698, June 1986.

[20] J. B. Weaver, Y. Xu, D. M. Healy, and J. R. Driscoll, "Wavelete encoded MR imaging," Magn. Reson. Med., vol. 24, pp. 275-287, 1992.

[21] J. Lu, D. M. Healy Jr., and J. B. Weaver, "Contrast enhancement of medical images using multiscale edge representation," Opt. Eng., pp. 1251-1261, July 1994.

[22] D. M. Healy Jr. and J. B. Weaver, "Two applications of wavelet transforms in MR imaging," IEEE Trans. Inform. Theory, vol. 38, pp. 840-868, Mar. 1992.

[23] J. B. Weaver, D. M. Healy Jr., H. Nagy, S. P. Poplack, J. Lu, T. Sauerland, and D. Langdon, "Classification of masses in digitized mammograms with features in the wavelet transform domain," in Proc. SPIE, Wavelet Applications, vol. 2242, H. H. Szu, Ed., 1994, pp. 704-710.

[24] C. W. Li, C. G. Zheng, and C. F. Tai, "Detection of ECG characteristic points using wavelet transforms," IEEE Trans. Biomed. Eng., vol. 42, pp. 21-28, Jan. 1995.

[25] J. S. Sahambi, S. N. Tandon, and R. K. P. Bhatt, "Using wavelet transform for ECG characterization," IEEE Eng. Med. Biol., vol. 16, pp. 77-83, 1997

[26] Z. Berman, "The uniqueness question of discrete wavelet maxima representation," System Research Center, University of Maryland, College Park, MD, Tech. Rep., TR 91-48, Apr. 1991.

[27] S. G. Mallat, "A complete signal representation from multiscale edges," New York University, NY, Tech. Rep. 483, Dec. 1989.

[28] B. M. Wu, P. W. F. Poon, F. H. Y. Chan, and F. K. Lam, "Microcomputer based system for long term analysis of activity profiles in rats," in Proc. BME'94 Hong Kong, 1994, pp. 355-358.

[29] J. Lu, J. B. Weaver, D. M. Healy, and J. Y. Xu, "Noise reduction with a multiscale edge representation and perceptual criteria," in Proc. IEEE Int. Symp. Time-Frequency and Time-Scale Analysis, Victoria, B.C., Oct. 1992 , pp. $555-558$.

[30] T. F. Elsmore, F. W. Hegge, P. Naitoh, T. Kelly, and D. Ryman, "WinCD: Complex demodulation for Windows," Chronobiol. Int., vol. 12, pp. 248-256, 1995. 


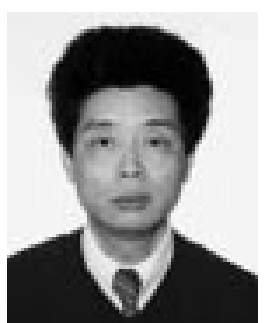

F. H. Y. Chan ( $\mathbf{M}^{\prime}$ 81) received the Ph.D.degree from Bristol University, Bristol, U.K.

He joined the Electrical and Electronic Engineering Department, The University of Hong Kong, Hong Kong, China, in 1976. He is currently Professor and Director of the University of Hong Kong Biomedical Engineering Research Centre, coordinating biomedical engineering research in the university. His research interest is in biomedical signal processing.

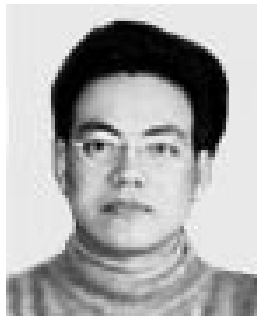

B. M. Wu (S'95) was born in China in $1962 . \mathrm{He}$ received the B.Sc. and M.Sc. degrees in electronic engineering from Chongqing University, Chongqing, China. He is currently working towards the Ph.D. degree in the Department of Electrical and Electronic Engineering at The University of Hong Kong, Hong Kong, China.

From 1988 to 1993 , he was Lecturer, and since 1995, Associate Professor, at Research Institute of Surgery, The Third Military Medical University, Chongqing, China. His main research interests include biomedical instrumentation and signal processing with special on evaluation, cure and rehabilitation techniques of central neural injury.

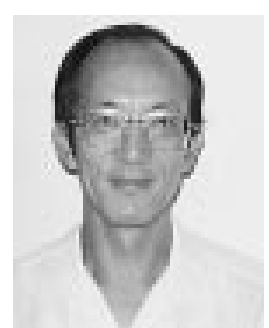

F. K. Lam received the B.Sc. (Eng.) from the University of Hong Kong, Hong Kong, China, and the M.Sc. and Ph.D. degrees from Loughborough University, Loughborough, Taiwan.

After working in Britain, he returned to Hong Kong University and now is Deputy Head of Department. His specialization is in signal processing and communication.

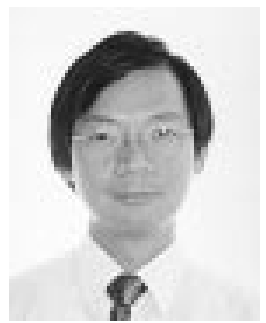

Paul W. F. Poon received the Ph.D. degree in neuroscience from Indiana University, Bloomington, IN.

$\mathrm{He}$ is currently Professor and Head, Department of Physiology, National Cheng Kung University, Tainan, Taiwan. His research interests include auditory physiology and biomedical signal processing.

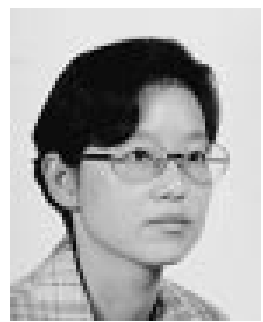

A. M. S. Poon received the M.B., B.S., M. Phil. and Ph.D. degrees from The University of Hong Kong, Hong Kong, China.

She joined the Department of Physiology, The University of Hong Kong in 1986 and engaged in basic biomedical research. Her current research interests include biorhythms and melatonin physiology. 